\title{
Shaping tin nanocomposites through transient local conversion reactions
}

\author{
Hans C. Hendrikse, ${ }^{1}$ Stivell Hémon-Charles,,${ }^{1,2}$ Lukas Helmbrecht, ${ }^{1}$ Eliane P. van Dam, ${ }^{1, \dagger}$ Erik \\ C. Garnett, ${ }^{1}$ Willem L. Noorduin ${ }^{1,3 *}$ \\ ${ }^{1}$ AMOLF, 1098 XG Amsterdam, The Netherlands \\ ${ }^{2}$ École Polytechnique l'Université de Nantes, 44035 Nantes, France \\ ${ }^{3}$ Van 't Hoff Institute for Molecular Sciences, University of Amsterdam, Amsterdam 1090 GD, The Netherlands \\ ${ }^{\dagger}$ current address: Department of Physical Chemistry II, Ruhr University Bochum, 44780 Bochum, Germany \\ ${ }^{*}$ Corresponding Author: noorduin@amolf.nl
}

\section{Supporting Information}

\section{Growth of $\mathrm{BaCO}_{3} / \mathrm{SiO}_{2}$ nanocomposites}

A substrate (e.g. $2 \times 2 \mathrm{~cm}$ slide of aluminum, stainless steel or silicon) was vertically positioned in a $100 \mathrm{~mL}$ beaker containing $\mathrm{BaCl}_{2}$ dihydrate $(74 \mathrm{mg}, 0,3 \mathrm{mmol})$ and $\mathrm{Na}_{2} \mathrm{SiO}_{3}(16 \mathrm{mg}, 0,13 \mathrm{mmol}$ ) dissolved in $15 \mathrm{~mL}$ of water. The reaction vessel was loosely covered with a Petri dish to allow $\mathrm{CO}_{2}$ from the air to diffuse slowly into the reaction mixture. Typical growth times ranged between 1.5-2.0 hours after which the substrate was removed from the solution and washed with deionized water. The structures were kept in this water for 5 minutes before converting them to $\mathrm{Sn}_{3}(\mathrm{OH})_{2} \mathrm{O}_{2}$ nanocomposites. The composition of the structures was determined using SEM, EDS and XRD (See Figure 1). The used reference for the XRD-diffractogram can be found in the Crystallography Open Database (COD 1000033).

\section{Conversion of $\mathrm{BaCO}_{3} / \mathrm{SiO}_{2}$ to $\mathrm{Sn}_{3}(\mathrm{OH})_{2} \mathrm{O}_{2} / \mathrm{SiO}_{2}$ nanocomposites}

Anhydrous $\mathrm{SnCl}_{2}$ (948 mg, $100 \mathrm{mM}$ ) was dissolved in $50 \mathrm{~mL}$ degassed water. Subsequently a substrate with $\mathrm{BaCO}_{3} / \mathrm{SiO}_{2}$ nanocomposites was placed in the solution for 30 seconds. The resulting nanocomposites were washed in two demineralized water baths followed by an acetone bath. After drying to the air, the converted nanocomposites were analyzed using SEM, XRD and EDS (See Figure 1). The used reference for the XRDdiffractogram can be found in the Crystallography Open Database (COD 9011384).

\section{Conversion to $\mathrm{Sn}_{3}(\mathrm{OH})_{2} \mathrm{O}_{2} / \mathrm{SiO}_{2}$ to $\mathrm{SnO}_{2} / \mathrm{SiO}_{2}$ nanocomposites}

A substrate containing $\mathrm{Sn}_{3}(\mathrm{OH})_{2} \mathrm{O}_{2} / \mathrm{SiO}_{2}$ nanocomposites was placed in the middle of a single zone horizontal tube furnace. The oven was heated to $200^{\circ} \mathrm{C}$ for 3 hours and subsequently heated to $600^{\circ} \mathrm{C}$ for 3 hours to convert the nanocomposites to $\mathrm{SnO}_{2} / \mathrm{SiO}_{2}$. SEM, EDS and XRD analysis was performed (Figure 3), where XRD confirmed formation of $\mathrm{SnO}_{2}$ (Reference used is COD 1534785).

\section{Conversion to $\mathrm{Sn}_{3}(\mathrm{OH})_{2} \mathrm{O}_{2} / \mathrm{SiO}_{2}$ to $\mathrm{CH}_{3} \mathrm{NH}_{3} \mathrm{SnX}_{3} / \mathrm{SiO}_{2}$ nanocomposites}

A substrate containing $\mathrm{Sn}_{3}(\mathrm{OH})_{2} \mathrm{O}_{2} / \mathrm{SiO}_{2}$ nanocomposites was placed in the middle of a single zone horizontal tube furnace. Subsequently an alumina boat containing methyl ammonium halide (bromine or iodine) was placed next to the substrate. The pressure inside the tube was lowered to below 0.1 mbar using a vacuum pump. The tube was then flushed with nitrogen until atmospheric pressure was reached. This process was repeated twice to purge the system of oxygen. Afterwards the temperature of the furnace was set to $120^{\circ} \mathrm{C}$ and a $30 \mathrm{sccm}$ 
flow of nitrogen was applied to increase the pressure to 70 mbar. After 30 minutes the reaction was stopped and the oven was passively cooled to room temperature. XRD, EDS and PL measurements (Figure 4) confirmed the formation of a tin halide perovskite (references used for the XRD measurements are COD 2104791 and 4335632 for the bromide and iodide perovskite respectively).

\section{Scanning Electron Microscope (SEM) and Energy-dispersive X-ray spectroscopy (EDS) characterization}

Samples were loaded into the SEM without applying a conductive metal coating. SEM images were obtained using a FEI Verios 460 equipped with an Everhart-Thornley detector (ETD) and a circular backscatter detector (CBS). The images were recorded at $5 \mathrm{kV}$ and $10 \mathrm{kV}$ respectively using a $100 \mathrm{pA}$ current in both instances. EDS was measured with the same electron microscope using an Oxford X-Max ${ }^{\mathrm{n}}$ energy dispersive X-ray spectrometer with an accelerating voltage of $20 \mathrm{kV}$ using a $100 \mathrm{pA}$ current.

\section{X-ray diffraction (XRD) characterization}

X-ray diffraction measurements were performed using a Bruker D2 Phaser (Bragg-Brentano geometry) using a $\mathrm{K}$-alpha $\mathrm{Cu}$ X-ray source with an emission energy of $8.0415 \mathrm{keV}$. The samples were mounted inside a chamber filled with ambient air at room temperature. All measurements were performed using a $1.0 \mathrm{~mm}$ beam knife to reduce unwanted scattering and optimize the low angle part of the diffractogram. A nickel filter was used to reduce the $20-30 \%$ contribution from the k-beta $\mathrm{Cu}$ X-ray source. A divergence slit of 0.1 or $1 \mathrm{~mm}$ was used to control the illuminated area of the sample, based on the spread of the material on the substrate. Diffracted Xrays were detected using a Lynxeye detector and were collected for at least 12 hours with a scan interval $(\Delta 2 \theta)$ of $0.01^{\circ}$.

\section{Grain size determination from XRD patterns}

The average grain size was determined using the Debye Scherrer Equation:

$$
\tau=\frac{\mathrm{K} \lambda}{\beta \cos \theta}
$$

where $\tau$ is the mean size of the crystalline domains, $\mathrm{K}$ is the shape factor and is estimated to be $0.9, \lambda$ is the wavelength of the $\mathrm{x}$-ray source (1.54060 $\AA$ ), $\beta$ is the line broadening at half height (fwhm) and $\theta$ is the Bragg angle. A complete list of all the crystal sizes can be found in Table S1.

\begin{tabular}{|l|l|}
\hline Crystal & Average Grain Size (nm) \\
\hline $\mathrm{BaCO}_{3}$ & 21 \\
\hline $\mathrm{Sn}_{3}(\mathrm{OH})_{2} \mathrm{O}_{2}$ & 33 \\
\hline $\mathrm{SnO}_{2}$ & 10 \\
\hline $\mathrm{CH}_{3} \mathrm{NH}_{3} \mathrm{SnBr}_{3}$ & 81 \\
\hline $\mathrm{CH}_{3} \mathrm{NH}_{3} \mathrm{SnI}_{3}$ & 90 \\
\hline
\end{tabular}

Table S1. Average grain sizes for all synthesized and converted crystals.

\section{Infrared Spectrometry (IR) Characterization}

IR spectra were recorded using a Bruker Vertex 80v FT-IR spectrometer equipped with an ATR module (Platinum ATR Diamond) by first measuring a background after 30 minutes to flush water vapor and $\mathrm{CO}_{2}$ out and then measuring the nanocomposites after a similar 30 minutes wait time.

\section{Photoluminescence and fluorescence microscopy characterization}

The photoluminescence measurements were performed on a WITec alpha 3000 SR. A $532 \mathrm{~nm}$ laser and a 405 nm laser (Thorlabs fiber coupled laser source S1FC405) were used to excite the iodide and bromide perovskite 
respectively. The signal was collected with a WITec UHTS 300 spectrometer and the corresponding detector (DV401A-BV-352). 


\section{Mechanism of Conversion}

Letting bulk conditions reach the nanocomposites

To demonstrate the importance of the temporal local reaction zone, a substrate with nanocomposites was kept in a conversion solution for 2 minutes. At this time, the $\mathrm{BaCO}_{3}$ has completely dissolved and the local reaction zone has disappeared. Without the locally increased $\mathrm{pH}$, the $\mathrm{Sn}_{3}(\mathrm{OH})_{2} \mathrm{O}_{2}$ inside the nanocomposites dissolves, leaving behind an empty $\mathrm{SiO}_{2}$ shell (Figure $\mathrm{S} 1$ ).

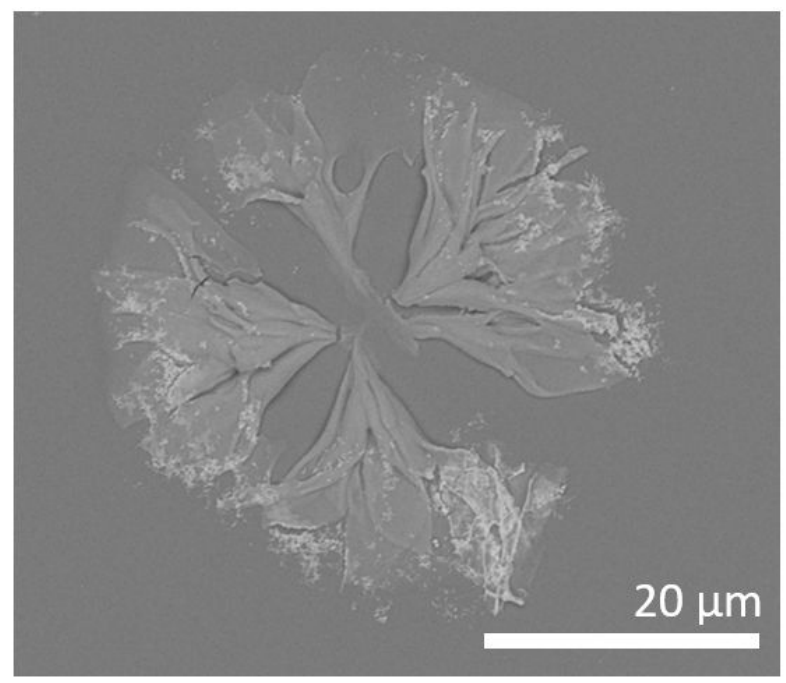

Figure S1. Nanocomposite kept in a $\mathrm{SnCl}_{2}$ conversion solution for 2 minutes. As the local reaction zone disappears around the nanocomposites after 30 seconds, this nanocomposite is depleted of its tin content.

\section{Increasing $\mathrm{pH}$ throughout the entire solution}

To discover the mechanism behind the conversion, we investigated the reaction of tin with water in the absence of the nanocomposites. Specifically, tin was dissolved in water and kept under nitrogen atmosphere, which resulted in a white precipitate forming after 30 minutes. This white precipitate was collected via vacuum filtration and analyzed using XRD. Comparing the obtained XRD diffractogram (figure S2, top) with AMCSD 0015835 confirmed the formation of Abhurite $\left(\mathrm{Sn}_{21} \mathrm{O}_{6}(\mathrm{OH})_{14} \mathrm{Cl}_{16}\right)$. Abhurite also formed under atmospheric conditions (figure S2, middle) at an accelerated rate (within 5 minutes), where an amorphous bump was observed in the XRD pattern. In contrast, we observed that during the conversion of nanocomposites hydroromarchite was formed rather than abhurite. Moreover, whereas in the bulk solution abhurite forms after more than 30 minutes, the nanocrystals within the composites convert into hydroromarchite within 30 seconds.

We hypothesize that this difference in precipitation behavior is caused by an increase in the local $\mathrm{pH}$ during the ion-exchange: Abhurite is stable at $\mathrm{pH} 2-5.5$, while hydroromarchite only precipitates above a $\mathrm{pH}$ of 3.5 (Edwards, R., Gillard, R. D. \& Williams, P. A. Mineral. Mag. 56, 221-226 (1992)), far above the solution's pH of 2.0. Direct observation of the local $\mathrm{pH}$ is however difficult. Therefore, to test this hypothesis, we deliberately increased the $\mathrm{pH}$ in the solution to induce the formation of hydroromarchite. To this aim we added $1 \mathrm{M} \mathrm{NaOH}$ (10 mL added to a $50 \mathrm{~mL}$ solution) at the start of the experiment. Precipitation formed almost instantly, comparable to the 30 second conversion we observe previously for hydroromarchite in the presence of the nanocomposites. Moreover, analyzing this precipitate with XRD (Figure S2, bottom) confirmed the formation of hydroromarchite (compared with COD 9011384) whereas no abhurite peaks were observed. Hence, increase of the $\mathrm{pH}$ indeed favors the formation of hydroromarchite over abhurite. In the case where nanocomposites are used, we find that the precipitate only forms locally in the nanocomposites. Thus, we concluded that the local basic conditions, induced by the dissolving of $\mathrm{BaCO}_{3}$, enables the hydroromarchite to form inside the nanocomposites. 


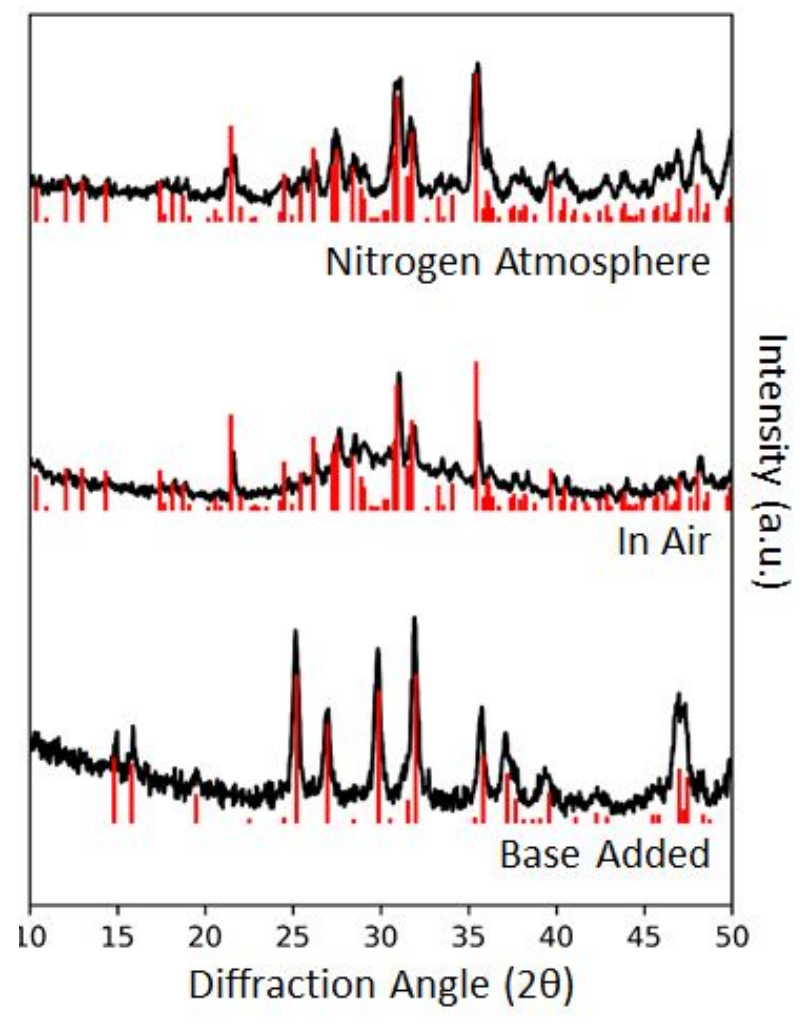

Figure S2. XRD of precipitate formed in water when dissolving $\mathrm{SnCl}_{2}$ under nitrogen (top), in air (middle) and after adding 1M NaOH (bottom). Reference spectra (red lines) are AMCSD 0015835 for abhurite, and COD 9011384 for hydroromarchite. 